\title{
A standard test case suite for two-dimensional linear transport on the sphere
}

\author{
P. H. Lauritzen ${ }^{1}$, W. C. Skamarock ${ }^{1}$, M. J. Prather ${ }^{2}$, and M. A. Taylor ${ }^{3}$ \\ ${ }^{1}$ National Center for Atmospheric Research, Boulder, Colorado, USA \\ ${ }^{2}$ Earth System Science Department, University of California, Irvine, California, USA \\ ${ }^{3}$ Sandia National Laboratories, Albuquerque, New Mexico, USA
}

Correspondence to: P. H. Lauritzen (pel@ucar.edu)

Received: 27 December 2011 - Published in Geosci. Model Dev. Discuss.: 30 January 2012

Revised: 22 May 2012 - Accepted: 25 May 2012 - Published: 28 June 2012

\begin{abstract}
It is the purpose of this paper to propose a standard test case suite for two-dimensional transport schemes on the sphere intended to be used for model development and facilitating scheme intercomparison. The test cases are designed to assess important aspects of accuracy in geophysical fluid dynamics such as numerical order of convergence, "minimal" resolution, the ability of the transport scheme to preserve filaments, transport "rough" distributions, and to preserve preexisting functional relations between species/tracers under challenging flow conditions.

The experiments are designed to be easy to set up. They are specified in terms of two analytical wind fields (one nondivergent and one divergent) and four analytical initial conditions (varying from smooth to discontinuous). Both conventional error norms as well as novel mixing and filament preservation diagnostics are used that are easy to implement. The experiments pose different challenges for the range of transport approaches from Lagrangian to Eulerian. The mixing and filament preservation diagnostics do not require an analytical/reference solution, which is in contrast to standard error norms where a "true" solution is needed. Results using the CSLAM (Conservative Semi-Lagrangian Multi-tracer) scheme on the cubed-sphere are presented for reference and illustrative purposes.
\end{abstract}

\section{Introduction}

A basic building block in any fluid dynamics solver is the transport operator that approximates the evolution of the bulk motion of a scalar. Despite intense research in transport schemes intended for global modeling on the sphere, only test 1 of the widely used test case suite by Williamson et al. (1992) seems to be the standard test, whereas other (newer) test cases are, in general, only optionally used. Test 1 in Williamson et al. (1992) is referred to as the solid-body advection test case, and the exact solution is simply the translation of the initial condition so that the center of the distribution follows a great circle. The flow field is non-divergent and does not challenge the transport operator with respect to deformation or divergence. In the last decade other nondivergent global test cases have been proposed such as static (Nair and Machenhauer, 2002) and moving vortices (Nair and Jablonowski, 2008) test cases that include deformation. Also for these tests the analytical solution is known at all times. Scheme developers do, in general, not publish results for all test cases and, perhaps more importantly, they often choose different parameter settings making it more difficult to compare results for different schemes. A purpose of this paper is to provide specific guidelines for test case setup in terms of parameters, resolution, time step, and diagnostics.

Perhaps more challenging, analytical wind fields were recently proposed by Nair and Lauritzen (2010). The Lagrangian fluid parcels follow complex trajectories (not great circles or small circles) making it harder to compute the analytical solution throughout the simulation. Following LeVeque (1996) the flow has a "time-reversing" component so that after one period the exact solution equals the initial condition. Half way through the simulation, however, the initial distributions are deformed into thin filaments and an "overlaid" translational flow transports the filaments as they deform. This problem is very challenging. A divergent wind 
field is proposed in Nair and Lauritzen (2010) as well which is in contrast to most idealized wind fields in the literature. The combination of both divergent and deformational flow constitutes a more realistic atmospheric/ocean transport than, for example, solid-body advection flow.

The idealized transport experiments listed above are all based on a single tracer, and accuracy is quantified in terms of conventional errors norms, i.e., quantifications of the differences between the analytical (exact) and numerically computed solutions. In some geophysical fluid dynamics problems, such as the transport of long-lived species in the stratosphere and aerosol-cloud interactions (Ovtchinnikov and Easter, 2009), it is not only important that individual species/tracers are transported accurately, but also the maintenance of pre-existing functional relations between species/tracers is important. Such models also cannot accept non-physical transport or redistribution of tracer that is not accompanied by resolved motion of air masses. Following Lagrangian fluid parcels, interrelations between tracers are conserved; however, any non-Lagrangian scheme will almost certainly perturb such relations. Nevertheless, Lagrangian schemes in realistic divergent flows must eventually combine parcels or create new ones, and that process will not likely preserve the relationships. Numerical errors that perturb pre-existing functional relations can resemble "real" mixing similar to what is observed in nature when mixing occurs (hereafter referred to as "real mixing") or the truncation errors can introduce unmixing (i.e., spurious mixing) (Thuburn and Mclntyre, 1997). A quantification and classification of mixing between interrelated tracers was recently proposed in Lauritzen and Thuburn (2012). For a more extensive overview of test cases for global models and desirable properties for transport schemes intended for atmospheric modeling, see, for example, the recent book chapter by Lauritzen et al. (2011).

The purpose of this paper is to propose a minimal and challenging test case suite with specific guidelines on the implementation and diagnostics, thereby facilitating intercomparison of schemes and establishing a benchmark data base for future developers. In the derivation of the tests, we sought to minimize the workload on transport scheme developers while evaluating their schemes in terms of a wide range of quantitative measures of accuracy considered important for geophysical fluid dynamics. Therefore, we assume that modelers have already tested their schemes in simpler settings such as with solid-body and static/moving vortices test cases and we do not repeat those tests here.

Almost any test case suite could be extended to include more tests that could provide more insights into specific aspects of accuracy particularly useful for some classes of schemes and applications. For example, Ullrich et al. (2012) found it insightful (for evaluating higher-order approximations to Lagrangian cell sides) to transport a constant using the initial condition wind field for the shallow water test case 3 of Williamson et al. (1992). Similarly, one could use the actual observed winds in test case 7 in Williamson et al. (1992) to generate more complex structures in the tracer field. To produce filaments that eventually become sub-gridscale in a context where the analytical solution is known, one may use the moving vortices test case (Nair and Jablonowski, 2008) and run it out further than the 12 days suggested in the test case description. The tests presented in this paper are not meant to be exhaustive and developers usually have preferred idealized tests specific for their application. It is the intent of this paper to present a minimal test bed based on just two analytic wind fields and four initial conditions that address a wide range of accuracy aspects, challenge both Lagrangian and Eulerian schemes with realistic conditions typical of 3-D flows, and make it straight forward to compare results from different schemes since we provide detailed instructions on test case setup and diagnostics. In doing so, we believe this test case suite provides new insights into accuracy beyond the much simpler and most widely used standard solid-body advection test case and associated standard error norms.

The test case proposal is organized as follows. In Sect. 2 the transport equation(s) is introduced followed by formulas for the analytical initial conditions and wind fields. The section is concluded with discretization details such as "definition" of resolution and maximum Courant numbers. The actual test case setup is given in Sect. 3 and it is divided into six categories designed to do the following: assess numerical convergence rates, "minimal" resolution, filament preservation, transport of discontinuous distributions, maintenance of pre-existing non-linear functional relations, and transport under divergent flow conditions. A list of algorithmic considerations/properties such as size of computational stencil, stability criterion, and number of function evaluations is put forward in Sect. 4. The paper is concluded with a summary of the test cases.

\section{General problem formulation}

\subsection{Transport equation(s)}

Consider a transport scheme that approximates the solution to the continuity equation for a passive (does not feed back on the flow) and inert (no sources or sinks) tracer:

$$
\frac{\partial(\rho \phi)}{\partial t}+\nabla \cdot(\rho \phi \boldsymbol{V})=0
$$

where $\rho$ is the fluid density, $\boldsymbol{V}$ is the two-dimensional flow velocity vector, and $\phi$ is the tracer mixing ratio per unit mass. Note that the discretized scheme is not necessarily based on the continuity equation written in flux-form as in Eq. (1) but could also be based on the advective form

$$
\frac{D \phi}{D t}=0
$$


or cell-integrated Lagrangian form

$$
\frac{D}{D t} \int_{A(t)} \rho \phi d A=0 \text {, }
$$

where $A(t)$ is a Lagrangian area and $D / D t$ is the total or material derivative. If tracer density $\rho \phi$ (flux-form Eq. 1 or cell-integrated Lagrangian Eq. 3 ) and not mixing ratio $\phi$ (advective form Eq. 2) is the prognostic variable, one needs to "extract" the mixing ratio $\phi$ from $\rho \phi$, which obviously requires the solution to the continuity equation for fluid density $\rho$ (see, e.g., Nair and Lauritzen 2010 for details). The mixing ratio $\phi$ is used for all diagnostics/analyses and not tracer density $\rho \phi$.

Define the discrete transport operator $\mathcal{T}$ that advances the numerical solution for $\phi$ in time:

$\phi_{k}^{n+1}=\mathcal{T}\left(\phi_{j}^{n}\right), \quad j \in \mathcal{H}$,

where $n$ is the time-level index, $k$ is the index for the grid cell/point, and $\mathcal{H}$ is the set of indices defining the "halo" or computational stencil required by $\mathcal{T}$. To compute the numerical solution $\phi_{k}^{n+1}$, an initial condition, a prescribed velocity field, and (if applicable) the solution to the continuity equation for fluid density $\rho$ are required.

\subsection{Initial conditions}

For fluid density (if needed) the initial condition is $\rho(t=$ $0)=1$ for all test cases. Four initial conditions for mixing ratio $\phi$ are used and defined in sub-sections below. We use one infinitely smooth $\left(C^{\infty}\right)$ initial condition, one quasi-smooth, one discontinuous, and one non-linearly correlated with the quasi-smooth initial condition. It may be argued that the discrete initial conditions should be as consistent as possible with the numerical method. For example, a finite-volume method is usually based on cell-averaged prognostic variables, and the initial condition in cell $k, \phi_{k}$, should be obtained by integrating the continuous initial condition $\phi$ over the $k$-th control volume. Similarly, methods that preserve and transport internal moments of the tracer distribution (e.g., Prather, 1986) should initialize these moments by integrating over the continuous starting distribution. Standard practice, however, is to use the value of the continuous initial condition evaluated at the centroid of the control volume as representative for the cell-averaged value, and higher-order moments (if applicable) are zero. It has been shown for finitevolume schemes that standard error norms may vary significantly when using point or cell-averaged values for initial conditions and for computing error norms (e.g., Lauritzen et al., 2010; Zerroukat et al., 2002). However, the conclusions drawn from the results are independent of the choice of exact solution (cell average versus grid-point value) as long as the schemes are compared with the same choice for exact solution in a consistent manner. Therefore, the initial condition and exact solution are based on grid-point values at the centroid of the grid cell for finite-volume methods and at quadrature/finite-difference points for basis-function/gridpoint methods.

All four initial conditions for $\phi$ are based on two distributions centered about $\left(\lambda_{i}, \theta_{i}\right), i=1,2$ :

$\left(\lambda_{1}, \theta_{1}\right)=(5 \pi / 6,0)$,

$\left(\lambda_{2}, \theta_{2}\right)=(7 \pi / 6,0)$,

where $\lambda$ is longitude and $\theta$ is latitude in radians. The distributions are symmetrically placed in the flow field to assess the symmetry of the numerically computed solution.

\subsubsection{Gaussian hills}

Smooth Gaussian surfaces/hills can be defined as follows:

$h_{i}(\lambda, \theta)=h_{\max } \exp \left\{-b\left[\left(\mathrm{X}-\mathrm{X}_{i}\right)^{2}+\left(\mathrm{Y}-\mathrm{Y}_{i}\right)^{2}+\left(\mathrm{Z}-\mathrm{Z}_{i}\right)^{2}\right]\right\}$,

where the height ${ }^{1}$ and width are determined by $h_{\max }=0.95$ and $b=5$, respectively (Levy et al., 2007). The absolute Cartesian coordinates $(\mathrm{X}, \mathrm{Y}, \mathrm{Z})$ and spherical $(\lambda, \theta)$ coordinates are related through

$(\mathrm{X}, \mathrm{Y}, \mathrm{Z})=(R \cos \theta \cos \lambda, R \cos \theta \sin \lambda, R \sin \theta)$,

where radius $R$ is the radius of the sphere. The coordinates for the center of the Gaussian distribution $\left(\mathrm{X}_{i}, \mathrm{Y}_{i}, \mathrm{Z}_{i}\right)$ are computed by inserting $\left(\lambda_{i}, \theta_{i}\right)$ into Eq. (8) and evaluating the right-hand side.

The Gaussian hills distribution is defined as the sum of the two Gaussian hills $h_{1}$ and $h_{2}$ (Eq. 7):

$\phi=\phi^{(g h)}(\lambda, \theta)=h_{1}(\lambda, \theta)+h_{2}(\lambda, \theta)$

and is graphically shown in Fig. 1a. Note that $\phi^{(g h)}$ is infinitely smooth $\left(C^{\infty}\right)$.

\subsubsection{Cosine bells}

Similarly, two symmetrically located cosine bells are defined as follows:

$h_{i}(\lambda, \theta)=\frac{h_{\max }}{2}\left[1+\cos \left(\pi r_{i} / r\right)\right] \quad$ if $\quad r_{i}<r$,

where the amplitude $h_{\max }=1$, base radius $r=R / 2$, and great circle distance between $(\lambda, \theta)$ and the center $\left(\lambda_{i}, \theta_{i}\right)$ is $r_{i}=r_{i}(\lambda, \theta)$, with

$r_{i}(\lambda, \theta)=R \arccos \left[\sin \theta_{i} \sin \theta+\cos \theta_{i} \cos \theta \cos \left(\lambda-\lambda_{i}\right)\right]$.

The initial condition $\phi$ consists of a background value $b$ and two cosine bells defined above:

$\phi=\phi^{(c b)}(\lambda, \theta)= \begin{cases}b+c h_{1}(\lambda, \theta) & \text { if } r_{1}<r, \\ b+c h_{2}(\lambda, \theta) & \text { if } r_{2}<r, \\ b & \text { otherwise, }\end{cases}$

where the background value is $b=0.1$ and amplitude $c=$ 0.9 , such that $\phi \in[0.1,1.0]$ (see Fig. 1b).

\footnotetext{
${ }^{1}$ note that Nair and Lauritzen (2010) used a $h_{\max }$ value of one which is different from the value used here
} 
(a) $\phi(t=0)$, Gaussian hills

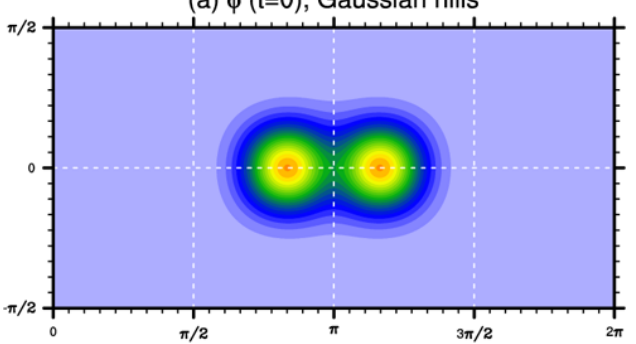

(c) $\phi(t=0)$, slotted cylinders

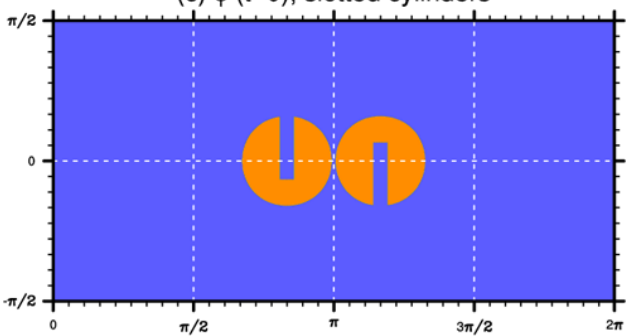

(b) $\phi(t=0)$, cosine bells

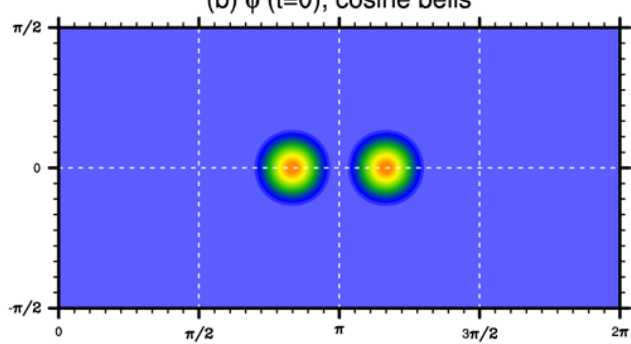

(d) $\phi(t=0)$, 'correlated' cosine bells

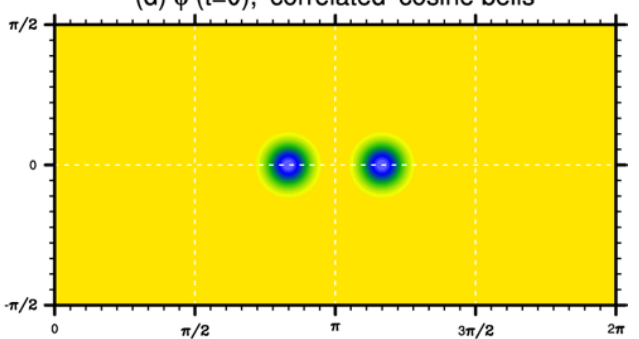

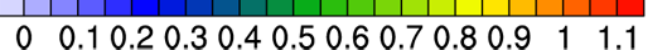

Fig. 1. Contour plots for the four initial conditions for mixing ratio $\phi$. (a) depicts the infinitely smooth $\left(\mathcal{C}^{\infty}\right)$ initial condition constructed from Gaussian surfaces, (b) the cosine bells initial condition which is $\mathcal{C}^{1}$, (c) the non-smooth slotted cylinders initial condition, and (d) is the initial condition, which is nonlinearly correlated with (b).

\subsubsection{Slotted cylinders}

For the discontinuous case, the double cosine-bells (Eq. 11) are replaced by slotted-cylinders (Zalesak, 1979):

$\phi=\phi^{(s c)}(\lambda, \theta)=$

$\left\{\begin{array}{l}c \text { if } r_{i} \leq r \text { and }\left|\lambda-\lambda_{i}\right| \geq r /(6 R) \text { for } i=1,2, \\ c \text { if } r_{1} \leq r \text { and }\left|\lambda-\lambda_{1}\right|<r /(6 R) \text { and } \theta-\theta_{1}<-\frac{5}{12} r / R, \\ c \text { if } r_{2} \leq r \text { and }\left|\lambda-\lambda_{2}\right|<r /(6 R) \text { and } \theta-\theta_{2}>\frac{5}{12} r / R, \\ b \text { otherwise, }\end{array}\right.$

where, again, the background value is $b=0.1$ and amplitude $c=1$ (see Fig. 1c).

\subsection{4 “Correlated" cosine bells}

An initial distribution that is nonlinearly "correlated" with the cosine bells initial condition is defined as

$\phi=\phi^{(c c b)}=\psi\left(\phi^{(c b)}\right)$.

The nonlinear functional relation is given by

$\psi(\chi)=a_{\psi} \chi^{2}+b_{\psi}$,

where

$a_{\psi}=-0.8 \quad$ and $\quad b_{\psi}=0.9$.

For a contour plot of the correlated cosine bells see Fig. 1d.

\subsection{Wind fields}

In this test case suite, we use two deformational wind fields: one non-divergent and one divergent. The components of the non-divergent velocity vector $\boldsymbol{V}(\lambda, \theta, t)$ and the stream function

$u=-\frac{\partial \psi}{\partial \theta}$,

$v=\frac{1}{\cos \theta} \frac{\partial \psi}{\partial \lambda}$,

are given by

$$
\begin{aligned}
u(\lambda, \theta, t)= & \frac{10 R}{T} \sin ^{2}\left(\lambda^{\prime}\right) \sin (2 \theta) \cos \left(\frac{\pi t}{T}\right) \\
& +\frac{2 \pi R}{T} \cos (\theta) \\
v(\lambda, \theta, t)= & \frac{10 R}{T} \sin \left(2 \lambda^{\prime}\right) \cos (\theta) \cos \left(\frac{\pi t}{T}\right), \\
\psi(\lambda, \theta, t)= & \frac{10 R}{T} \sin ^{2}\left(\lambda^{\prime}\right) \cos ^{2}(\theta) \cos \left(\frac{\pi t}{T}\right) \\
& -\frac{2 \pi R}{T} \sin (\theta),
\end{aligned}
$$

respectively, where $\lambda^{\prime}=\lambda-2 \pi t / T$. In non-dimensional units $T=5$ and $R=1$. An "Earth"-like dimensionalization of the wind fields may be obtained by setting $T=12$ days and $R=6.3712 \times 10^{6} \mathrm{~m}$. Schemes based on characteristics (typically Lagrangian schemes) may use the algorithm given 
(a) $\phi(t=T / 2)$, Gaussian hills

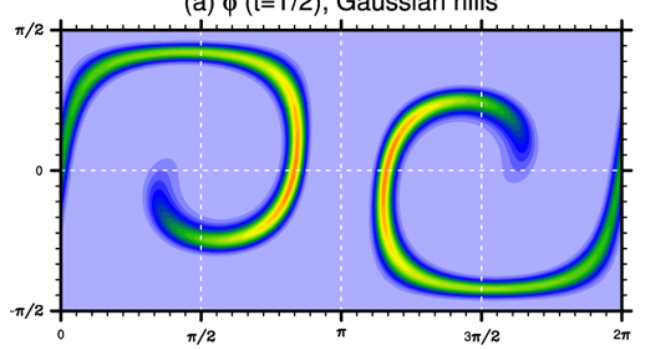

(c) $\phi(t=T / 2)$, slotted cylinders

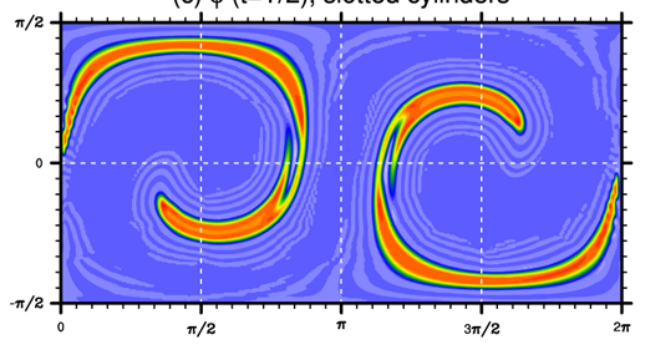

(b) $\phi(\mathrm{t}=\mathrm{T} / 2)$, cosine bells

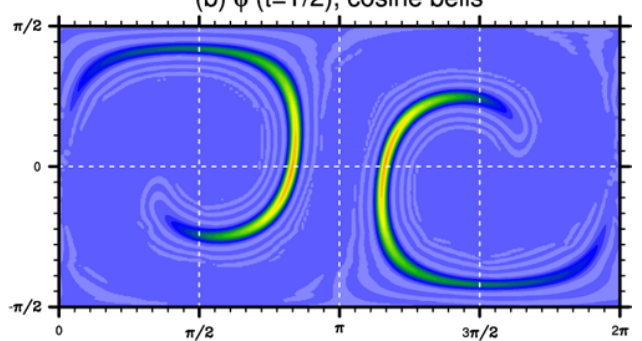

(d) $\phi(t=T / 2)$, 'correlated' cosine bells

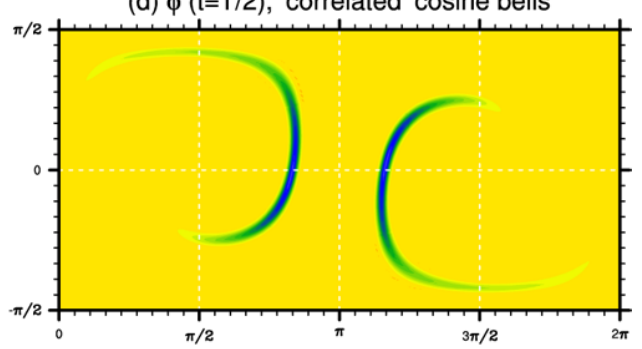

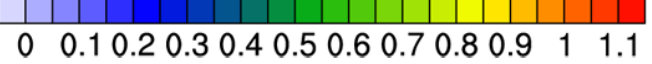

Fig. 2. Same as Fig. 1 but for the numerical solution at $t=T / 2$ using CSLAM with a time step $\Delta t=T / 120$ and resolution of $\Delta \lambda=1.5^{\circ}$.

in Nair and Lauritzen (2010) for the computation of parcel trajectories.

When either of the initial conditions given in Sect. 2.2 are transported by the non-divergent wind field, they are deformed into thin filaments half way through the simulation and these are simultaneously being transported eastward by the solid-body component of the flow (see Figs. 2 and 3). At maximum deformation, the filaments are approximately $10^{\circ}$ wide when using the cosine bells initial condition.

To challenge schemes under divergent flow conditions, we use the following wind field (Nair and Lauritzen, 2010, their case-3 with a "constant background wind field"):

$$
\begin{aligned}
u(\lambda, \theta, t)= & -5 \frac{R}{T} \sin ^{2}\left(\frac{\lambda^{\prime}}{2}\right) \sin (2 \theta) \cos ^{2}(\theta) \cos \left(\frac{\pi t}{T}\right) \\
& +\frac{2 \pi R}{T} \cos (\theta), \\
v(\lambda, \theta, t)= & \frac{5}{2} \frac{R}{T} \sin \left(\lambda^{\prime}\right) \cos ^{3}(\theta) \cos \left(\frac{\pi t}{T}\right),
\end{aligned}
$$

where $R$ and $T$ have the same values as for the non-divergent velocity field. The non-divergent flow field (Eqs. 18 and 19) is used for all tests except the test described in Sect. 3.6, for which the divergent winds are used (Eqs. 21 and 22).

The exact solution for all tests is known at $t=T$, and it is identical to the initial condition

$\phi(t=T)=\phi(t=0)$.

We do not have an exact solution throughout the simulation when using either the non-divergent or divergent flow field.
Note that the first part of the simulation $(t \in[0, T / 2])$ is typical of atmospheric/oceanographic flows in that features collapse to smaller scales, whereas the second part $(t \in[T / 2, T])$, in which the reverse occurs, is atypical of atmospheric/oceanographic flows though convenient for obtaining a problem with an exact solution. The background mean flow ensures that errors, in general, do not cancel when the deformational part of the flow reverses.

\subsection{Discretization details}

We specify resolution in terms of average grid-spacing in degrees at the Equator of the sphere $\Delta \lambda$. For methods based on quadrature methods, the "average resolution" should be specified in terms of mean distance between quadrature points. We define the (maximum) Courant number as

$$
\mathrm{CN}=\frac{\Delta t U_{\max }}{\Delta \lambda\left(\frac{\pi}{180^{\circ}}\right)}
$$

where $\Delta t$ is the time step and $U_{\max }$ is the maximum zonal wind speed. For the non-divergent flow, the non-dimensional and dimensional ("Earth") $U_{\max }$ are given by

$U_{\max } \approx 3.26$ and $U_{\max } \approx 100.07 \mathrm{~ms}^{-1}$,

respectively.

This definition of Courant number obviously does not emphasize local Courant numbers (in particular for nonisotropic grids); it is defined to facilitate comparison of maximum Courant numbers across discretization grids.

The time step $\Delta t$ should be a "typical/practical" time step for performing tracer transport with the scheme in question. 
(a) $\phi(t=T / 2)$, Gaussian hills

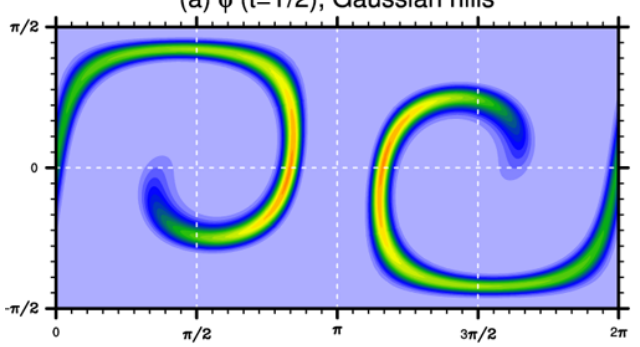

(c) $\phi(t=T / 2)$, slotted cylinders

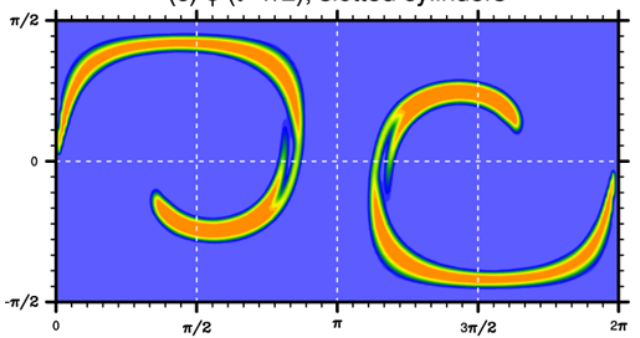

(b) $\phi(t=T / 2)$, cosine bells

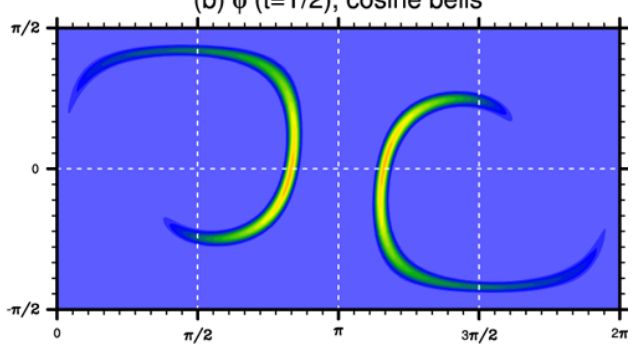

(d) $\phi(t=T / 2)$, 'correlated' cosine bells

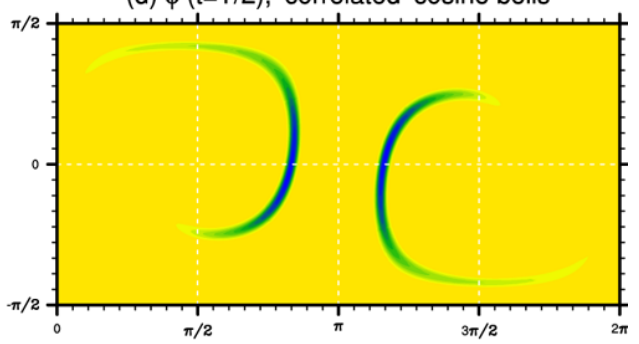

$\begin{array}{lllllllllllll}0 & 0.1 & 0.2 & 0.3 & 0.4 & 0.5 & 0.6 & 0.7 & 0.8 & 0.9 & 1 & 1.1\end{array}$

Fig. 3. Same as Fig. 2 but for CSLAM with a shape-preserving reconstruction function filter (see Lauritzen et al., 2010 for details on the filter).

However, investigating accuracy as a function of time step is also of interest. For example, if the transport scheme permits long time steps (Courant number $>1$ ), it is advised to run the tests with an "Eulerian" time step (Courant number $\leq 1$ ) as well.

Often limiters/filters are applied to render the numerically computed solution physically realizable. These may be shape-preserving, positive definite, monotone, and/or nonoscillatory limiters/filters. If schemes have a limiter/filter option, the tests should be run both without and with limiters/filters. If the limiters/filters are formulated in terms of tunable parameters for minima and maxima, the test case suite should be run with parameters used in "practical" applications of the scheme. For example, a tunable parameter (if applicable) should be set for a minimum value of mixing ratio that is zero, although in this test case suite the background value is non-zero..

Accuracy is assessed in terms of several diagnostics. First of all, we use standard error norms that are defined in Appendix A. These require knowledge of the "true" (analytic) solution and are therefore computed at time $t=T$ when the true solution is known. Secondly, we use recently proposed mixing diagnostics (Sect. 3.5; Appendix B and C) as well as a novel filament preservation diagnostic (Sect. 3.3). As these diagnostics do not require an analytical solution, we compute them at the time of maximum deformation $(t=T / 2)$ before the flow "reversal", which is less physical.

For reference purposes, we provide results using the CSLAM (Conservative Semi-Lagrangian Multi-tracer) scheme (Lauritzen et al., 2010) on the cubed-sphere grid. The CSLAM configuration used here is described in detail in Nair and Lauritzen (2010).

\section{Test cases}

The diagnostics/test cases are designed to assess the following:

1. numerical order of convergence,

2. "minimal" resolution,

3. ability of the transport scheme to preserve filaments,

4. ability of the transport scheme to transport "rough" distributions,

5. ability of the transport scheme to preserve pre-existing functional relations between tracers,

6. ability of transport scheme to deal with divergent flows (Nair and Lauritzen, 2010).

Each category is discussed in separate sections below.

\subsection{Numerical order of convergence: Gaussian hills}

This test is designed to assess the formal (or "optimal") order of convergence of the scheme under quasi-realistic flow conditions on the sphere. This is done as follows. Standard error norms using the Gaussian hills initial condition Eq. (9) 

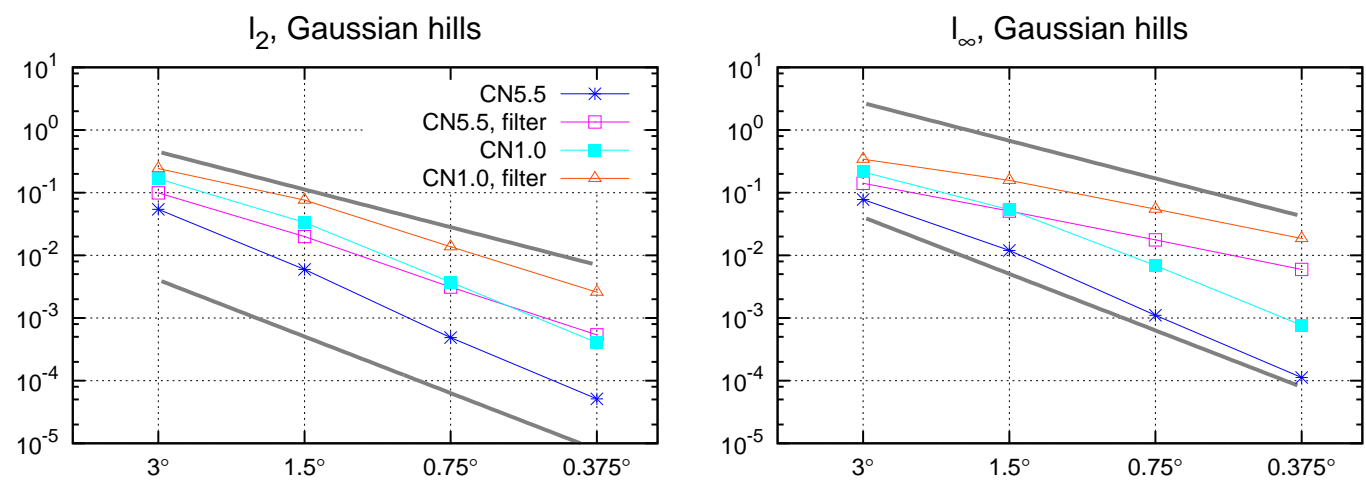

Fig. 4. Convergence plots for $\ell_{2}$ (first column) and $\ell_{\infty}$ (second column), respectively, computed with CSLAM with Gaussian hills initial conditions. The keys with "CN5.5" and "CN1.0" refer to simulations using a non-dimensional time step of $T / 120$ and $T / 600$, respectively. The keys with the word filter in them refer to simulations using a shape-preserving filter. The upper and lower heavy lines on each plot correspond to the slopes of second- and third-order convergence rates, respectively.

and non-divergent wind field (Eqs. 18 and 19) at resolutions ranging from approximately $\Delta \lambda=3^{\circ}$ to $\Delta \lambda=0.3^{\circ}$ for fixed Courant number are computed. The choice of resolutions should provide enough data points on a "convergence plot" (e.g., $\log \left(\ell_{2}\right)$ as a function of $\left.\log (N)\right)$ in the resolution interval of interest, to generate a "credible" estimate of numerical rate of convergence. For example, the following resolutions could be used: $\Delta \lambda=3^{\circ}, 1.5^{\circ}, 0.75^{\circ}, 0.375^{\circ}$. The runs should be performed without any limiting/filtering and (if applicable) also with limiters/filters enforcing shape-preservation, monotonicity and/or non-oscillatoriness in the numerically computed solution.

These simulations with infinitely smooth (Gaussian hills) initial conditions should provide a numerical estimate of the "optimal" numerical convergence rate of the scheme. A way to estimate numerical (empirical) convergence rates $\mathcal{K}_{2}$ and $\mathcal{K}_{\infty}$, for $\ell_{2}$ and $\ell_{\infty}$ respectively (see Fig. 4), is to perform a least-squares linear regression of the form (Harris et al., 2010):

$\log \left(\ell_{i}\right)=\mathcal{A}_{i}-\mathcal{K}_{i} \log (\Delta \lambda), \quad i=2, \infty$.

\section{2 "Minimal" resolution $\Delta \lambda_{m}$ : cosine bells}

In many geophysical fluid dynamics applications using stateof-the-art physical parameterization packages, increases in horizontal resolution come at significant computational cost. It is therefore of interest to assess the absolute error in addition to convergence rates. To do that we repeat the experiment described in Sect. 3.1 but with cosine bells initial condition (11) to find the "minimal" resolution. We define the "minimal" resolution $\Delta \lambda_{m}$ as the $\Delta \lambda$-value for which $\ell_{2}$ is approximately 0.033 , when using an unlimited scheme and the cosine bells Eq. (11) initial condition (the Courant number used for defining $\Delta \lambda_{m}$ should be one typically used by the scheme). A convergence plot can conveniently be used to find the "minimal" resolution by finding the intersection

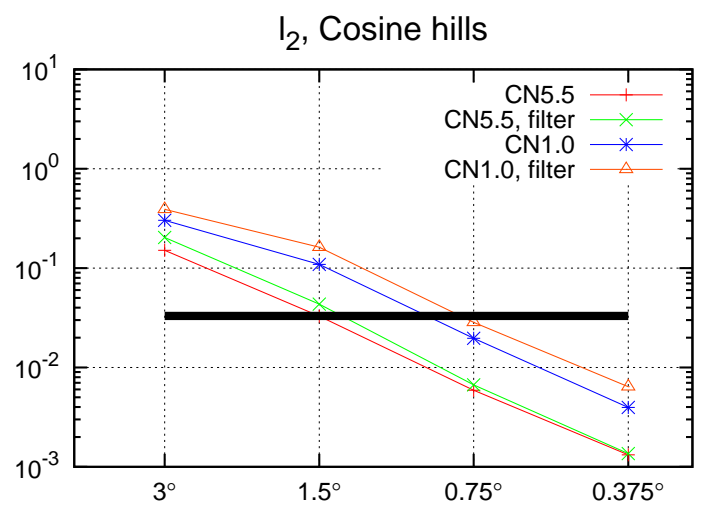

Fig. 5. Convergence plot for $\ell_{2}$ computed with CSLAM with cosine bells initial conditions. The keys are as in Fig. 4. The heavy line is $\ell_{2}=0.033$ and is used to define "minimal" resolution.

between the horizontal line $\ell_{2}=0.033$ and the convergence curve for $\ell_{2}$ (see Fig. 5). The quasi but not infinitely smooth initial conditions (Cosine bells instead of Gaussian hills) are used in order to challenge the schemes with respect to weak non-smoothness.

The "minimal" resolution $\Delta \lambda_{m}$ will be used in the remaining test cases. The choice of threshold for $\Delta \lambda_{m}$ is based on results for CSLAM (a resolution for which the thin filaments are marginally resolved). The "minimal" resolution (as defined here) for CSLAM is $\Delta \lambda=1.5^{\circ}$ and $\Delta \lambda \approx 1^{\circ}$ when using a time step of $T / 120$ (maximum Courant number is approximately 5.2) and $T / 600$ (maximum Courant number is approximately 1.0).

\section{3 "Filament" preservation diagnostic $\ell_{f}$ : cosine bells}

Realistic flows often deform distributions into thin filaments that, in general, are challenging to represent by Eulerian and semi-Lagrangian transport schemes that use a fixed grid in 
(a) $1^{\text {st }}$-order CSLAM

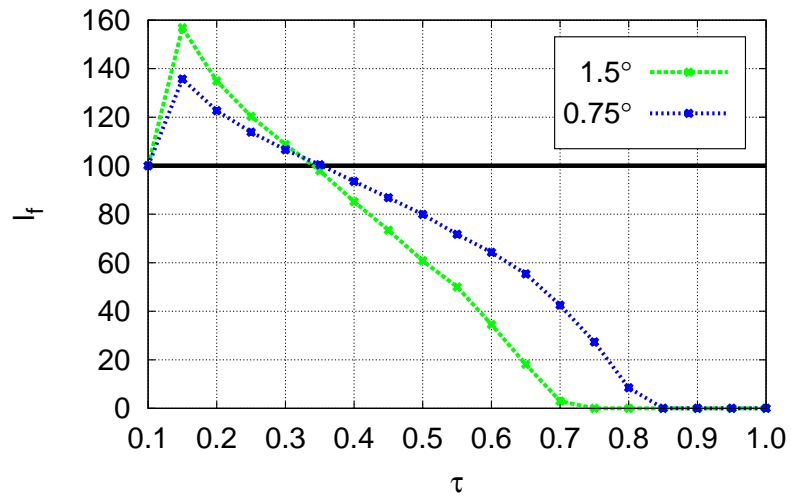

(b) $3^{\text {rd }}$-order CSLAM

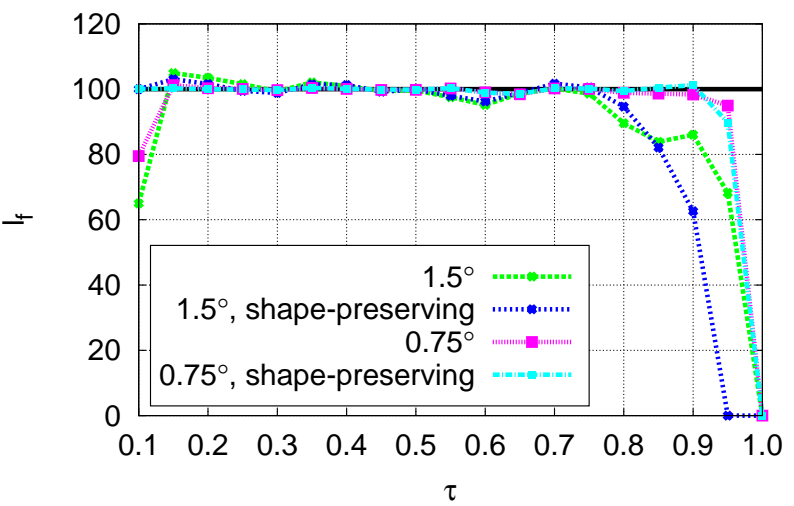

Fig. 6. Filament diagnostics $\ell_{f}(t=T / 2)$ as a function of threshold value $\tau$ for different configurations of the CSLAM scheme with Courant number 5.5. (a) first-order version of CSLAM at $\Delta \lambda=1.5^{\circ}$ and $\Delta \lambda=0.75^{\circ}$, and (b) third-order version of CSLAM with and without shape-preserving filter at resolutions $\Delta \lambda=1.5^{\circ}$ and $\Delta \lambda=0.75^{\circ}$.

space (e.g., Behrens et al., 2000). A measure of how well a transport scheme preserves gradients (in particular thin filaments) is relevant for many tracer applications (e.g., transport of long-lived tracers such as chemical species in the stratospheric vortices). Filaments are created when material surfaces stretch and gradients increase. When the thickness of the filaments reach the scale at which molecular diffusion (or some other diffusive process) becomes important, the filaments are no longer preserved but gradients are eroded. For the flow and initial conditions considered here, the filaments should, for all practical purposes, be preserved by the transport scheme, as the physical scale of the filaments is approximately $10^{\circ}$ at maximum deformation. We do therefore not assess how transport schemes represent the filament erosion process that appears in nature since those "diffusive" processes take place at scales several magnitudes below $10^{\circ}$. If such processes are of interest, we suggest to use the moving vortices test case of Nair and Jablonowski (2008) and extend the simulation time so that the filaments are stretched to a level where such processes are important and/or change the parameters in the Nair and Lauritzen (2010) flow field to increase the amount of deformation (see, e.g., Kent et al., 2012).

The "filament" preservation diagnostic is formulated as follows. Define $A(\tau, t)$ as the spherical area for which the spatial distribution of the $\operatorname{tracer} \phi(\lambda, \theta)$ satisfies

$\phi(\lambda, \theta) \geq \tau$,

at time $t$, where $\tau$ is the threshold value. For a non-divergent flow field and a passive and inert tracer $\phi$, the area $A(\tau, t)$ is invariant in time.

The discrete definition of $A(\tau, t)$ is

$A(\tau, t)=\sum_{k \in \mathcal{G}} \Delta A_{k}$ where $\Delta A_{k}$ is the spherical area for which $\phi_{k}$ is representative and $\mathcal{G}$ is the set of indices

$\mathcal{G}=\left\{k \in(1, \ldots, K) \mid \phi_{k} \geq \tau\right\}$,

where $K$ is the number of grid cells. For Eulerian finitevolume schemes, $\Delta A_{k}$ is the area of the $k$ th control volume. For Eulerian grid-point schemes, a control volume for which the grid-point value is representative must be defined. Similarly, for fully Lagrangian schemes based on point values (parcels), control volumes for which the point values are representative must be defined. Note that the "control volumes" should span the entire domain without overlaps or "cracks" between them.

Define the filament preservation diagnostic

$\ell_{f}(\tau, t)= \begin{cases}100.0 \times \frac{A(\tau, t)}{A(\tau, t=0)} & \text { if } A(\tau, t=0) \neq 0, \\ 0.0, & \text { otherwise. }\end{cases}$

For infinite resolution (continuous case) and a nondivergent flow, $\ell_{f}(\tau, t)$ is invariant in time: $\ell_{f}(\tau, t=0)=$ $\ell_{f}(\tau, t)=100$ for all $\tau$. At finite resolution, however, the filament diagnostic even for an exact scheme should not necessarily be preserved since the solution must be truncated to the discrete grid. That said, usually the numerical truncation errors are much larger than the grid truncation error at least at moderate resolutions.

The experimental setup is as in Sect. 3.2, i.e., uses the non-divergent wind field (Eqs. 18 and 19) and cosine bells initial condition Eq. (11). At half time, $t=T / 2$; the filament preservation diagnostic $\ell_{f}(\tau, t=T / 2)$ is computed at 19 equi-distant discrete intervals $(\tau=0.10,0.15,0.20$, $0.25, \ldots, 0.95,1.00$ ) without and (if applicable) with limiters/filters at $\Delta \lambda=1.5^{\circ}, \Delta \lambda=0.75^{\circ}$ as well as at the "minimal" resolution $\Delta \lambda=\Delta \lambda_{m}$. The filament diagnostic should be computed as a function of $\tau \in[0.1,1.0]$ (see Fig. 6). The 
(a) $\phi(t=T / 2)$, no filter/limiter

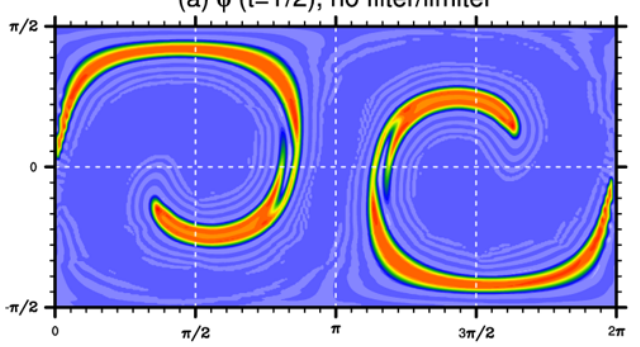

(c) $\phi(t=T / 2)$, shape-preserving filter

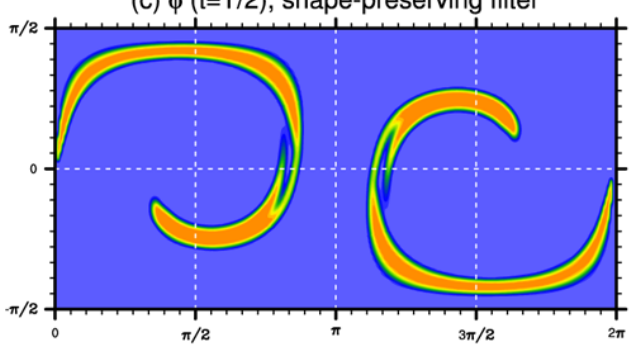

(b) $\phi$ ( $\mathrm{t}=\mathrm{T})$, no filter/limiter

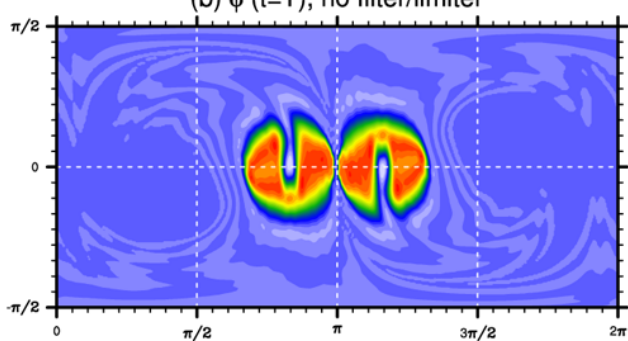

(d) $\phi(t=T)$, shape-preserving filter

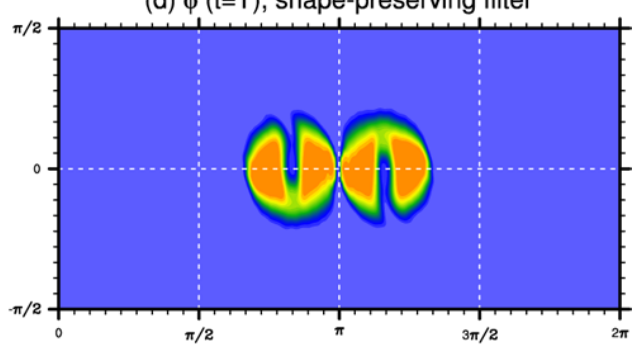

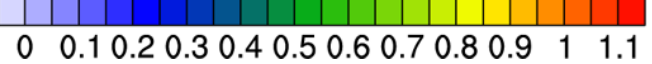

Fig. 7. Contour plot of the CSLAM numerical solution $\phi$ at resolution $\Delta \lambda=1.5^{\circ}$ and time step $T / 120$ using the slotted-cylinders initial condition at time $t=T / 2$ (a and $\mathbf{c}$ ) and $t=T$ (b and $\mathbf{d}$ ) using no filter/limiter (a and b) and a shape-preserving filter (c and d). The standard error norms for the unfiltered/unlimited solution are $\ell_{2}=0.24, \ell_{\infty}=0.79, \phi_{\min }=-0.19$, and $\phi_{\max }=0.15$, and for the shape-preserving solution they are $\ell_{2}=0.26, \ell_{\infty}=0.80, \phi_{\min }=0.0$, and $\phi_{\max }=-4.34 \times 10^{-3}$.

threshold value for which $\ell_{f}(t=T / 2)$ is less than, for example, 80 is a measure for how well filaments are preserved.

Numerical diffusion will tend to decrease $\ell_{f}$ for large $\tau$ values (maxima decrease) and increase $\ell_{f}$ for low $\tau$ values (gradients are "smeared"). An "extreme" situation is shown in Fig. 6a where $\ell_{f}$ is plotted as a function of $\tau$ for the highly diffusive first-order version of CSLAM. This much improves when using the higher-order version of CSLAM (Fig. 6b). Note that the non-shape-preserving versions of CSLAM produce values of $\ell_{f}$ less than 100.0 for low threshold values $(\tau<0.1)$. This also indicates an error in tracer transport due to undershoots $(\phi<0.1)$, which are not represented in the $\ell_{f}$ diagnostic.

\subsection{Transport of "rough" distribution: slotted-cylinders}

To challenge shape-preserving filters/limiters (if applicable), we use discontinuous initial conditions, i.e., standard error norms for the simulated solution at $t=T$ using the slotted cylinders initial condition and non-divergent winds (Eqs. 18 and 19) are computed using the transport scheme without and (if applicable) with limiters/filters at resolutions $\Delta \lambda=1.5^{\circ}$, $\Delta \lambda=0.75^{\circ}$ as well as at the "minimal" resolution $\Delta \lambda_{m}$. Contour plots of the solution at $t=T / 2$ and $t=T$ (Fig. 7) using a contour interval of 0.05 in the range $[0.0: 1.1]$ are shown.

\subsection{Preservation of pre-existing functional relation: cosine bells and correlated cosine bells}

In the tests described in the previous sections, the accuracy is assessed in a single-tracer setup. Now we consider two tracers that are both advected by the same non-divergent flow field (Eqs. 18 and 19). The initial conditions for the two tracers are the cosine bells initial condition (Eq. 11) and correlated cosine bells (Eq. 13), respectively (see Fig. 1b and d). The mixing ratios of the two tracers are referred to as $\chi$ and $\xi$. Following Lagrangian parcels, any functional relation between tracers should mathematically be preserved at all times, and hence any deviation from the pre-existing functional relation between the tracers is essentially numerical error introduced by the transport scheme. Note that the "ideal" scheme could be a scheme that does not exactly preserve pre-existing functional relations, but for which the numerical errors are less than physical diffusive processes in nature.

In any case transport schemes should not disrupt functional relations in unphysical ways. Numerical errors that perturb such relations essentially introduce mixing or unmixing between the tracers. Lauritzen and Thuburn (2012) provide a discussion of the physical importance of transport schemes not disrupting tracer interrelationships in

\footnotetext{
2 a bug was identified in the code used for computing mixing diagnostics in Lauritzen and Thuburn (2012).
} 
(a) CSLAM, $1^{\text {st }}$-order, $1.5^{\circ}$

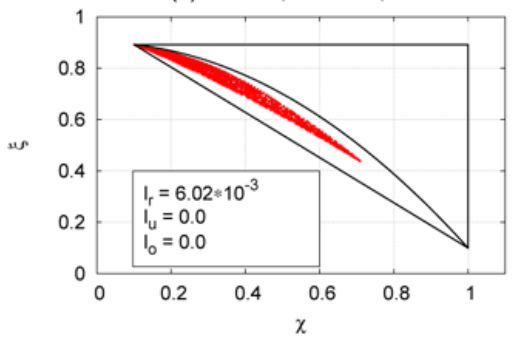

(d) CSLAM, $1^{\text {st }}$-order, $0.75^{\circ}$

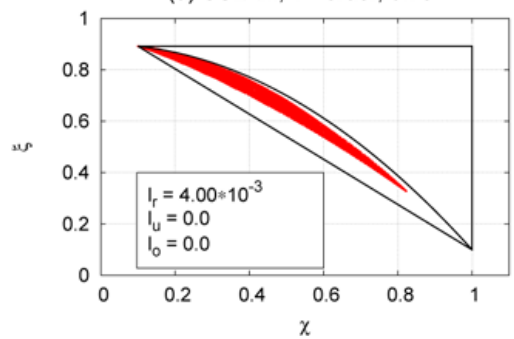

(b) CSLAM, no filter, $1.5^{\circ}$

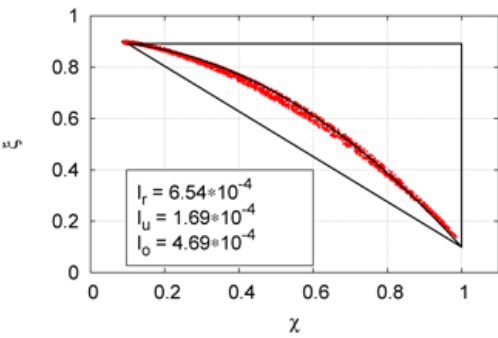

(e) CSLAM, no filter, $0.75^{\circ}$

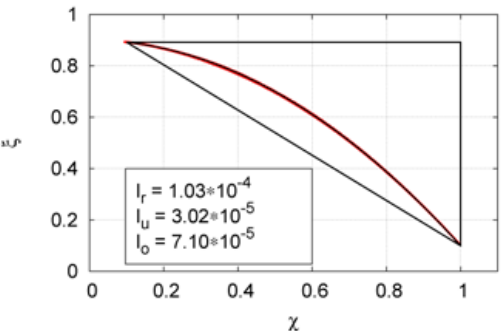

(c) CSLAM, shape-preserving filter, $1.5^{\circ}$
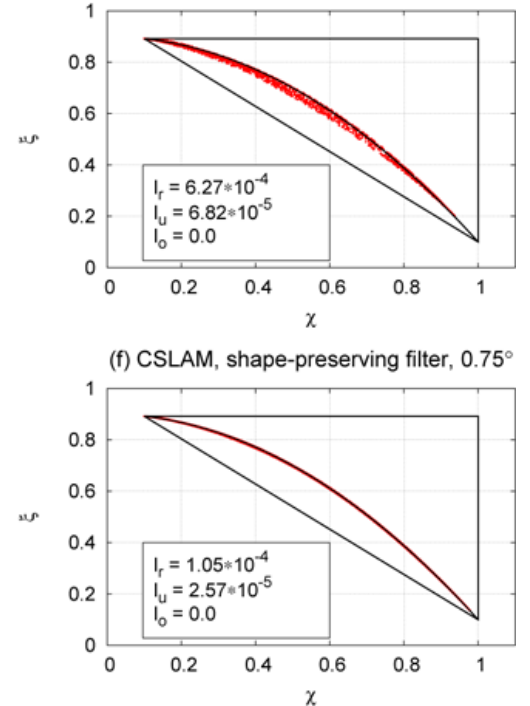

Fig. 8. Scatter plots at $t=T / 2$ for two non-linearly correlated species/tracers based on cosine bells initial conditions using first-order version of CSLAM (a and d), standard CSLAM based on bi-parabolic reconstruction functions (b and e) and standard CSLAM with a shapepreserving filter ( $\mathbf{c}$ and $\mathbf{f}$ ). First and second row use $\Delta \lambda=1.5^{\circ}$ and $\Delta \lambda=0.75^{\circ}$ resolutions, respectively. The solid lines mark the boundaries between the areas used to classify the numerical mixing. On each plot the mixing diagnostics $\ell_{r}, \ell_{u}$ and $\ell_{o}$ are given. $^{2}$

unphysical ways with special focus on non-linear chemistry. The numerical errors that perturb pre-existing functional relations between tracers will be referred to as numerical mixing or simply mixing in this paper (one could equally well use terminology such as tracer variance dissipation instead of mixing). In nature such processes that change the correlation between two tracers come about through diffusive processes, and, for reactive tracers, through chemical reactions between tracers. The purpose of this test is to quantify the amount of mixing and the physical realizability of the mixing that a scheme introduces through truncation errors. Note that preserving correlations are, however, no guarantee for accuracy, as one may design schemes that satisfy tracer interrelations but are otherwise inaccurate; as formulated by Thuburn and Mclntyre (1997), "shaping two tracer fields the same way does not imply shaping them the right way".

Scatter plots, where tracer 1 ( $\chi$ using cosine bells initial condition) and tracer 2 ( $\xi$ using correlated cosine bells initial condition) are plotted against each other, are used to quantify the numerical mixing or unmixing introduced by the scheme (see Fig. 8). As discussed in Thuburn and Mclntyre (1997), no Eulerian scheme can exactly preserve pre-existing nonlinear relations between two tracers, and hence scatter points $\left(\chi_{k}, \xi_{k}\right)$ will, in general, deviate from the pre-existing functional relation curve $\psi$ as the simulation evolves. The way in which the scatter points deviate from the non-linear $\psi$-curve has implications for the character of the numerical mixing that the transport scheme introduces. For this test it is crucial that features collapse in scale, and we therefore consider scatter plots using prognosed mixing ratios at half time $(t=T / 2)$ when the initial condition has deformed into thin filaments and collapsed to smaller scales compared to the initial condition.

Following Lauritzen and Thuburn (2012), the numerical mixing (deviation of scatter points $\left(\chi_{k}, \xi_{k}\right)$ from $\psi$-curve) is classified into three categories:

- "Real" mixing: numerical mixing that resembles "real" mixing (e.g., Thuburn and Mclntyre, 1997) when scatter points move to the concave side of $\psi$. All other deviations from the pre-existing functional curve follow spurious unmixing, which is accounted for in two separate categories.

- "Range-preserving" unmixing: numerical unmixing within the range of the initial data, i.e., scatter points are shifted to the convex side of the pre-existing functional relation or below the convex hull but not outside the range of the initial data.

- Overshooting (or equivalently expanding range unmixing): numerical unmixing that is not "range-preserving" unmixing, which for this specific test case setup is $(\chi, \xi) \notin[0.1,1.0]^{2}$.

The deviation of the scatter points from the $\psi$-curve is quantified in terms of a normalized shortest distance between $\left(\chi_{k}, \xi_{k}\right)$ and the $\psi$-curve referred to as $d_{k}$. For the specific parabolic non-linear correlation function used here (Eq. 14), the normalized distance function $d_{k}$ is given in Appendix B.

The three diagnostics that quantitatively account for numerical mixing that resembles "real" mixing, 
(a) $\phi(t=T / 2)$, no filter/limiter

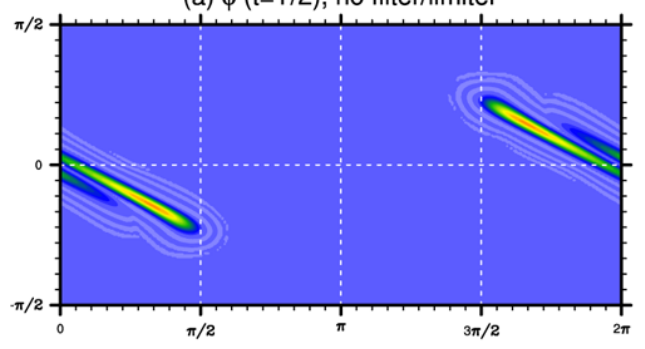

(c) $\phi(t=T / 2)$, shape-preserving filter

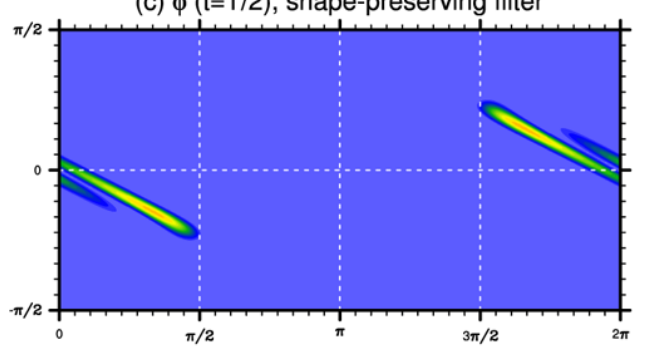

(b) $\phi(t=T)$, no filter/limiter

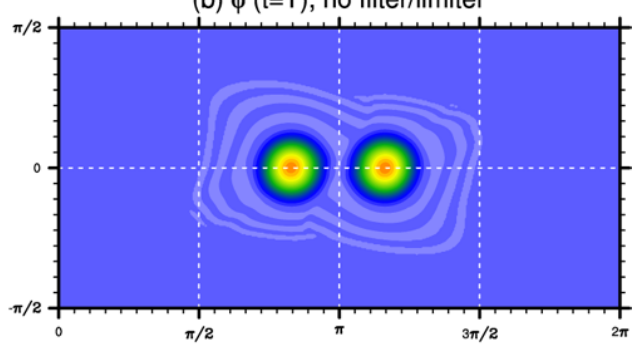

(d) $\phi(t=T)$, shape-preserving filter

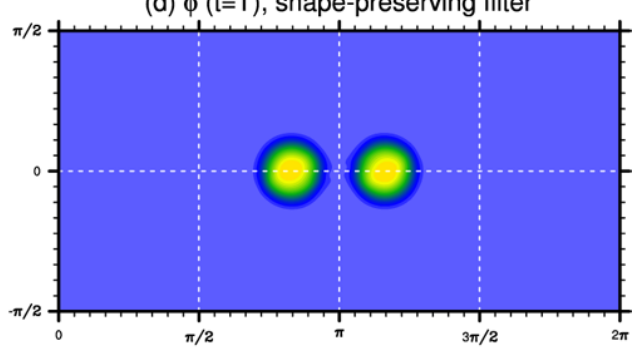

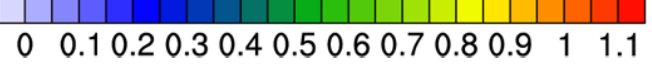

Fig. 9. Plotted as in Fig. 7 but for the divergent flow field $\left(\Delta t=T / 120\right.$, resolution $\Delta \lambda=1.5^{\circ}$, and maximum Courant number is approximately 3.2).

"range-preserving" unmixing and overshooting are referred to as $\ell_{r}, \ell_{u}$, and $\ell_{o}$, respectively, and are formally defined in Appendix C. For more discussion on numerical mixing and the physical reasoning behind the classification of the mixing, see Lauritzen and Thuburn (2012). Note that knowledge of the exact solution is not needed for the computation of the mixing diagnostics.

Using the non-divergent flow field, we compute the mixing diagnostics $\left(\ell_{r}, \ell_{u}, \ell_{o}\right)$ half way through the simulation $t=T / 2$ using two non-linearly correlated tracer distributions $\chi=\phi^{(c b)}$ and $\xi=\phi^{(c c b)}$ as initial conditions (cosine bells and correlated cosine bells) at resolutions $\Delta \lambda=1.5^{\circ}$, $\Delta \lambda=0.75^{\circ}$ and $\Delta \lambda_{m}$ using the unlimited and (if applicable) shape-preserving scheme. The scatter plots, that is, the mixing ratio of one tracer (with cosine bells initial conditions) against the other (with non-linearly correlated cosine bells initial condition) at these resolutions, are shown in Fig. 8.

It is noted that transport schemes can be designed to preserve linear pre-existing functional relations, i.e., a scheme will preserve linear correlations between species/tracers if the transport operator $\mathcal{T}$ satisfies

$\mathcal{T}(A \phi+B)=A \mathcal{T}(\phi)+B \mathcal{T}(1)=A \mathcal{T}(\phi)+B$,

where $A$ and $B$ are constants (Lin and Rood, 1996; Thuburn and Mclntyre, 1997). It is assumed that schemes have already been tested with respect to preservation of linear correlations without and (if applicable) with limiters/filters.

\subsection{Transport under divergent flow conditions: cosine bells}

Most idealized test cases are formulated in terms of nondivergent wind fields. Since realistic flows are divergent it should be demonstrated that the transport operator can handle divergent winds. We repeat the experiment described in Sect. 3.4 using the divergent wind field (see Eqs. 21 and 22), cosine bells initial conditions Eq. (11), and the same time steps. Solutions using CSLAM are shown on Fig. 9.

Error norms for unlimited CSLAM at $\Delta \lambda=1.5^{\circ}$ resolution with $\Delta t=120 / T$ are $\ell_{2}=1.90 \times 10^{-2}, \ell_{\infty}=3.22 \times$ $10^{-2}, \phi_{\min }=-2.33 \times 10^{-2}$, and $\phi_{\max }=-1.45 \times 10^{-2}$. Similar for shape-preserving CSLAM, we obtain: $\ell_{2}=4.22 \times$ $10^{-2}, \ell_{\infty}=0.11, \phi_{\min }=0.0, \phi_{\max }=-0.13$.

\section{Algorithmic considerations}

Overall, algorithmic considerations or properties are documented. By algorithmic considerations we refer to general properties/characteristics of the scheme that usually impact scheme implementation, stability, and data-flow. Below is a non-exhaustive list of algorithmic considerations/properties:

- size of halo/stencil $\mathcal{H}$ used to update a cell/grid-point value;

- for multi-step time-stepping algorithms, specify number of stages (right-hand side evaluations); 
- number of integral/functional evaluations (if applicable) per time-stepping stage;

- maximum Courant number for which the transport scheme is stable;

- amount of information (if any) that can be re-used to transport additional tracers (multi-tracer efficiency).

\section{Summary}

Below is a summary of the proposed test case suite. In terms of implementation work, only two flows fields and four initial conditions are needed. The accuracy is assessed using traditional/conventional error norms as well as novel filamentpreservation and mixing diagnostics. For convenience the standard error norms $\ell_{i}, i=2, \infty, \phi_{\min }$ and $\phi_{\max }$ are computed at the end of the simulation $t=T$ when the exact solution is known (i.e., it equals the initial condition). All mixing diagnostics $\ell_{i}, i=r, u, o$, and the filament diagnostic $\ell_{f}$ (they do not require knowledge of the analytical solution to the transport equation) are computed half way through the simulation at $t=T / 2$ when the fields are most deformed.

For the non-divergent flow field (Eqs. 18 and 19), the following experiments and associated diagnostics are proposed:

1. numerical order of convergence showing convergence plots and computing numerical convergence rates $\mathcal{K}_{i}$ for $\ell_{i}, i=2, \infty$, for the resolution range approximately $\Delta \lambda=3^{\circ}$ to $\Delta \lambda=0.3^{\circ}$ using Gaussian initial conditions for the unlimited and (if applicable) shape-preserving scheme (Sect. 3.1),

2. "minimal" resolution computing "minimal" resolution $\Delta \lambda_{m}$ for which $\ell_{2} \approx 0.033$ using cosine bells initial condition for the unlimited and (if applicable) shapepreserving scheme (Sect. 3.2),

3. "filament" preservation plotting the filament preservation diagnostic $\ell_{f}$ (at $t=T / 2$ ) using the cosine bells initial condition for the unlimited and (if applicable) shape-preserving scheme at resolutions $\Delta \lambda=$ $1.5^{\circ}, \Delta \lambda=0.75^{\circ}$, and $\Delta \lambda=\Delta \lambda_{m}$ as a function of $\tau$ (Sect. 3.3),

4. "rough" distribution showing contour plots (using contour interval of 0.1 starting at 0.0 ) at times $t=T / 2, T$ and computing $\ell_{i}, i=2, \infty, \phi_{\min }$ and $\phi_{\max }$ at resolutions $\Delta \lambda=1.5^{\circ}, \Delta \lambda=0.75^{\circ}$, and $\Delta \lambda=\Delta \lambda_{m}$ for the slotted-cylinder initial conditions (12) using the unlimited and (if applicable) the shape-preserving scheme (Sect. 3.4), and

5. mixing diagnostics showing scatter plots and computing mixing diagnostics $\ell_{i}, i=r, u, o$, for the two nonlinearly correlated tracers based on cosine bells for the unlimited and (if applicable) shape-preserving scheme at resolutions $\Delta \lambda=1.5^{\circ}, \Delta \lambda=0.75^{\circ}$, and $\Delta \lambda=\Delta \lambda_{m}$ (Sect. 3.5).

Using the divergent flow field (Eqs. 21 and 22),

6. Divergent flow conditions computing standard error norms $\ell_{i}, i=2, \infty, \phi_{\min }$ and $\phi_{\max }$ at resolutions $\Delta \lambda=1.5^{\circ}, \Delta \lambda=0.75^{\circ}$, and $\Delta \lambda=\Delta \lambda_{m}$ using cosine bells initial conditions using the same time steps as used for test 4 above (Sect. 3.6) using the unlimited and (if applicable) shape-preserving scheme.

In addition to accuracy diagnostics under different flow conditions and using different initial conditions, we report on algorithm properties/characteristics by

7. Algorithmic considerations specifying size of computational stencil, number of right-hand side evaluations in multi-stage time-stepping schemes, stability criteria, and amount of information that can be re-used for each additional prognostic tracer.

Some results for the CSLAM scheme are given in this paper. Full results for the benchmark tests using CSLAM and a dozen of other state-of-the-art transport schemes are reported on in a separate publication (Lauritzen et al., 2012). Fortran code to compute mixing diagnostics $\left(\ell_{i}, i=r, u, o\right)$ and the filament diagnostic $\ell_{f}$ is available in the Supplement. Also Gnuplot scripts to compute convergence rates $\mathcal{K}_{i}, i=2, \infty$, as well as NCL (NCAR Command Language) scripts for plotting are available in the supplemental material. Modelers are especially encouraged to use the same color Table for contour plotting as used in the NCL script provided in the Supplement to facilitate visual scheme intercomparison.

\section{Appendix A}

\section{Standard error measures}

If $\phi=\phi(\lambda, \theta, t)$ is the transported mixing ratio field, then global normalized standard errors are defined by Williamson et al. (1992):

$$
\begin{aligned}
\ell_{2} & =\left[\frac{I\left[\left(\phi-\phi_{T}\right)^{2}\right]}{I\left[\left(\phi_{T}\right)^{2}\right]}\right]^{1 / 2}, \\
\ell_{\infty} & =\frac{\max _{\forall \lambda, \theta}\left|\phi-\phi_{T}\right|}{\max _{\forall \lambda, \theta}\left|\phi_{T}\right|}, \\
\phi_{\max } & =\frac{\max _{\forall \lambda, \theta}(\phi)-\max _{\forall \lambda, \theta}\left(\phi_{T}\right)}{\Delta \phi_{0}}, \\
\phi_{\min } & =\frac{\min _{\forall \lambda, \theta}(\phi)-\min _{\forall \lambda, \theta}\left(\phi_{T}\right)}{\Delta \phi_{0}},
\end{aligned}
$$

where $\phi_{T}$ and $\phi_{0}$ are, respectively, the exact/analytical solution, and its initial value, $\Delta \phi_{0}$, is the difference between 


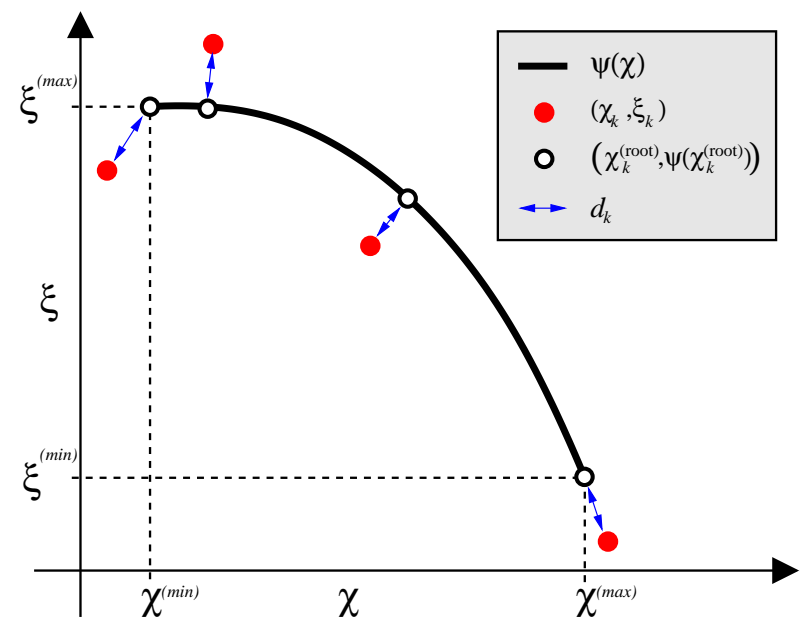

Fig. B1. A schematic of the "minimum" distance function $d_{k}$ (leftright arrows) for different correlation points $\left(\chi_{k}, \xi_{k}\right)$ (filled circles). $\left(\chi_{k}^{(\psi)}, \psi\left(\chi_{k}^{(\psi)}\right)\right.$ (unfilled circle) is the point on the pre-existing functional curve (thick line) that is nearest, in a normalized sense, to $\left(\chi_{k}, \xi_{k}\right)$.

maximum and minimum value of the initial condition, and the global integral $I$ is defined as follows:

$I(\phi)=\frac{1}{4 \pi} \int_{0}^{2 \pi} \int_{-\pi / 2}^{\pi / 2} \phi(\lambda, \theta, t) \cos \theta d \lambda d \theta$.

\section{Appendix B}

\section{Definition of distance function $d_{k}$}

The "minimum" distance function $d_{k}$ is defined as the minimal normalized Euclidean distance between the scatter point $\left(\chi_{k}, \xi_{k}\right)$ and the pre-existing functional relation curve $(\chi, \psi(\chi))$ within the range of the initial condition

$d_{k}=L_{k}\left(\chi_{k}^{(\psi)}\right)$,

where

$\chi_{k}^{(\psi)}=\min \left[\max \left(\chi^{(\min )}, \chi_{k}^{(\mathrm{root})}\right), \chi^{(\max )}\right]$

constrains the shortest distance to the initial condition interval $\left[\chi^{\mathrm{min}}, \chi^{\mathrm{max}}\right]$, and the normalized distance function is given by

$L_{k}(\chi)=\sqrt{\left(\frac{\chi_{k}-\chi}{R_{\chi}}\right)^{2}+\left(\frac{\xi_{k}-\psi(\chi)}{R_{\xi}}\right)^{2}}$,

where

$$
\begin{aligned}
& R_{\chi}=\chi^{(\max )}-\chi^{(\min )} \\
& R_{\xi}=\xi^{(\max )}-\xi^{(\min )}=\psi\left(\xi^{(\max )}\right)-\psi\left(\xi^{(\min )}\right)
\end{aligned}
$$

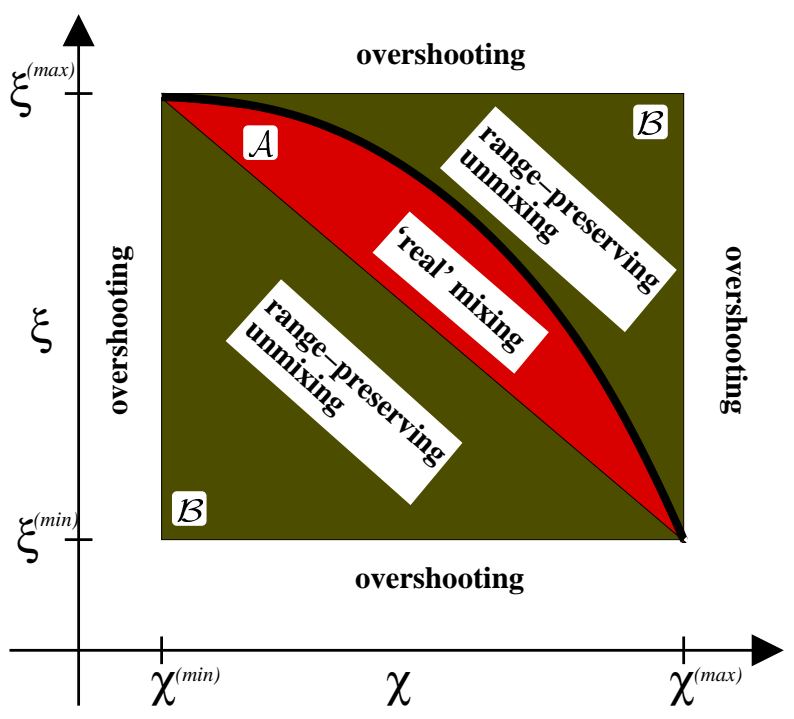

Fig. C1. A schematic of the classification of numerical mixing. If a scatter point is located in the area labeled with $\mathcal{A}$ (mathematically defined in Eq. C2), it is categorized as "real" mixing. Similarly, for the area labeled with $\mathcal{B}$ (defined in Eq. C4), it is categorized as range-preserving unmixing. The remaining part of the domain is referred to as overshooting. The thick solid line is the pre-existing non-linear functional relation curve. See text or Lauritzen and Thuburn (2012) for details.

For this particular test case setup $R_{\chi}=0.9, R_{\xi}=0.792$, and the "root" $\chi_{k}^{(\text {root })}$ is given by

$\chi_{k}^{(\text {root })}=c_{k}+\frac{1}{c_{k}}\left(\frac{13}{75}-\frac{5}{12} \xi_{k}\right)$,

where

$c_{k}=\frac{1}{60}\left[65340 \chi_{k}+12 \sqrt{12\left(125 \xi_{k}-52\right)^{3}+29648025 \chi_{k}^{2}}\right]^{1 / 3}$.

\section{Appendix C}

\section{Numerical mixing diagnostics}

For the two-tracer test (Sect. 3.5), three mixing diagnostics are used and defined below (Lauritzen and Thuburn, 2012).

\section{C1 Mixing that resembles "real" mixing}

"Real" mixing is defined as numerical mixing that resembles "real" mixing, in that values are shifted to the concave side of the pre-existing functional relation only (area $\mathcal{A}$ on Fig. C1):

$\ell_{r}=\frac{1}{A} \sum_{k=1}^{K} \begin{cases}d_{k} \Delta A_{k}, & \text { if }\left(\chi_{k}, \xi_{k}\right) \in \mathcal{A}, \\ 0, & \text { else, }\end{cases}$ 
where $K$ is the total numbers of cells/points in the domain, $\Delta A_{k}$ is the spherical area of grid cell $k$ and $A$ is the total area of the domain, $A=\sum_{k=1}^{K} \Delta A_{k}$. The distance function $d_{k}$ is the shortest normalized distance between the numerically computed scatter point $\left(\chi_{k}, \xi_{k}\right)$ and the pre-existing functional curve within the range of the initial conditions. For the quadratic functional relation $\psi$ given in Eq. (14) with coefficients (15), the explicit formula for $d_{k}$ is given in Appendix B. The domain $\mathcal{A}$ ("convex hull") is shown on Fig. $\mathrm{C} 1$ and is mathematically defined as

$\mathcal{A}=\left\{(\chi, \xi) \mid \chi_{k} \in\left[\chi^{(\min }, \chi^{(\max )}\right]\right.$ and $\left.\mathcal{F}\left(\chi_{k}\right) \leq \xi_{k} \leq \psi\left(\chi_{k}\right)\right\}$,

where $\mathcal{F}$ is the straight line that connects $\left(\chi^{(\min )}, \xi^{(\max )}\right)$ and $\left(\chi^{(\max )}, \xi^{(\min )}\right)$. Any other mixing (i.e., scatter points not in $\mathcal{A}$ ) is numerical unmixing that is accounted for in two distinct diagnostics defined next.

\section{C2 “Range-preserving” unmixing}

"Range-preserving" unmixing is defined as numerical unmixing within the range of the initial data, i.e., scatter points are shifted to the convex side of the pre-existing functional relation or below the convex hull but not outside the range of the initial data:

$\ell_{u}=\frac{1}{A} \sum_{k=1}^{K} \begin{cases}d_{k} \Delta A_{k}, & \text { if }\left(\chi_{k}, \xi_{k}\right) \in \mathcal{B} \\ 0, & \text { else }\end{cases}$

where $\mathcal{B}$ is the dark shaded areas in Fig. $\mathrm{C} 1$ defined by

$$
\begin{array}{r}
\mathcal{B}=\left\{(\chi, \xi) \mid\left(\chi_{k}, \xi_{k}\right) \in\left[\chi^{(\min }, \chi^{(\max )}\right] \times\left[\xi^{(\min }, \xi^{(\max )}\right]\right. \\
\text { and } \left.\left(\chi_{k}, \xi_{k}\right) \notin \mathcal{A}\right\} .
\end{array}
$$

Note that the shape-preservation constraint is not necessarily enough to guarantee $\ell_{u}=0$, since the scheme must be semilinear and monotone according to Harten (1983) to guarantee $\ell_{u}=0$ (Thuburn and Mclntyre, 1997). Only first-order schemes will satisfy these constraints (Godunov, 1959).

\section{C3 Overshooting}

Overshooting (or equivalently expanding range unmixing) is defined as unmixing that is not accounted for in the $\ell_{r}$ and $\ell_{u}$ diagnostic:

$\ell_{o}=\frac{1}{A} \sum_{k=1}^{K} \begin{cases}d_{k} \Delta A_{k}, & \text { if }\left(\chi_{k}, \xi_{k}\right) \notin \mathcal{A} \text { and }\left(\chi_{k}, \xi_{k}\right) \notin \mathcal{B}, \\ 0, & \text { else. }\end{cases}$

For a shape-preserving scheme, $\ell_{o}=0$.

The mixing diagnostics are "mutually exclusive" in the sense that for a particular scatter point $\left(\chi_{k}, \xi_{k}\right)$, a non-zero value of the distance function $d_{k}$ is only added to one of the diagnostic functions, so

$\ell_{r}+\ell_{o}+\ell_{u}=\frac{1}{A} \sum_{A} d_{k} \Delta A_{k}$

\section{Supplementary material related to this article is available online at: http://www.geosci-model-dev.net/5/ 887/2012/gmd-5-887-2012-supplement.zip.}

Acknowledgements. The many useful discussions with participants at the 2011 NCAR workshop on transport schemes are gratefully acknowledged. The lead author thanks R. D. Nair for many insightful discussion and acknowledges partial supported by the DOE BER Program under award DE-SC0001658. We thank the two reviewers (one anonymous and J. Behrens) for their encouraging reviews of this paper. Also thanks to D. Calhoun for identifying a coding error in the mixing diagnostics.

The National Center for Atmospheric Research is sponsored by the National Science Foundation.

Edited by: H. Garny

\section{References}

Behrens, J., Dethloff, K., Hiller, W., and Rinke, A.: Evolution of small-scale filaments in an adaptive advection model for idealized tracer transport, Mon. Weather Rev., 128, 2976-2982, 2000.

Godunov, S. K.: A difference scheme for numerical computation of discontinuous solutions of equations in fluid dynamics, Math. Sb., 47, 271-306, also: Cornell Aero. Lab. translation, 1959.

Harris, L. M., Lauritzen, P. H., and Mittal, R.: A Flux-form version of the Conservative Semi-Lagrangian Multi-tracer transport scheme (CSLAM) on the cubed sphere grid, J. Comput. Phys., 230, 1215-1237, doi:10.1016/j.jcp.2010.11.001, 2010.

Harten, A.: On the symmetric form of systems of conservation laws with entropy, J. Comput. Phys., 49, 151-164, 1983.

Kent, J., Jablonowski, C., Whitehead, J., and Rood, R. B.: Downscale Cascades in Tracer Transport Test-Cases: An Intercomparison of the Dynamical Cores in Community Atmosphere Model CAM5, Geosci. Model Dev. Discuss., submitted, 2012.

Lauritzen, P. H. and Thuburn, J.: Evaluating advection/transport schemes using scatter plots and numerical mixing diagnostics, $\mathrm{Q}$ J. Roy. Meteorol. Soc., 138, 906-918, doi:10.1002/qj.986, 2012.

Lauritzen, P. H., Nair, R. D., and Ullrich, P. A.: A conservative semi-Lagrangian multi-tracer transport scheme (CSLAM) on the cubed-sphere grid, J. Comput. Phys., 229, 1401-1424, doi:10.1016/j.jcp.2009.10.036, 2010.

Lauritzen, P. H., Ullrich, P. A., and Nair, R. D.: Atmospheric transport schemes: desirable properties and a semi-Lagrangian view on finite-volume discretizations, in: Numerical Techniques for Global Atmospheric Models, edited by: Lauritzen, P. H., Nair, R. D., Jablonowski, C., and Taylor, M., Lect. Notes Comput. Sci. Eng., Springer, 80, 185-250, doi:10.1007/978-3-642-11640-7_8, 2011. 
Lauritzen, P. H., Andronova, N., Bosler, P. A., Calhoun, D., Enomoto, T., Dong, L., Dubey, S., Guba, O., Hansen, A. B., Jablonowski, C., Juang, H.-M. H., Kaas, E., Kent, J., Müller, R., Penner, J. E., Prather, M. J., Reinert, D., Skamarock, W. C., Sørensen, B., Taylor, M. A., Ullrich, P. A., and White III, J. B.: A standard test case suite for 2D linear transport on the sphere: results from 17 state-of-the-art schemes, Geosci. Model Dev. Discuss., in preparation, 2012.

LeVeque, R. J.: High-resolution conservative algorithms for advection in incompressible flow, SIAM J. Numer. Anal., 33, 627-665, 1996.

Levy, M. N., Nair, R. D., and Tufo, H. M.: High-order Galerkin method for scalable global atmospheric models, Comput. Geosci., 33, 1022-1035, 2007.

Lin, S. J. and Rood, R. B.: Multidimensional Flux-Form SemiLagrangian Transport Schemes, Mon. Weather Rev., 124, 20462070, 1996.

Nair, R. D. and Jablonowski, C.: Moving Vortices on the Sphere: A Test Case for Horizontal Advection Problems, Mon. Weather Rev., 136, 699-711, 2008.

Nair, R. D. and Lauritzen, P. H.: A Class of Deformational Flow Test Cases for Linear Transport Problems on the Sphere, J. Comput. Phys., 229, 8868-8887, doi:10.1016/j.jcp.2010.08.014, 2010.

Nair, R. D. and Machenhauer, B.: The Mass-Conservative CellIntegrated Semi-Lagrangian Advection Scheme on the Sphere, Mon. Weather Rev., 130, 649-667, 2002.
Ovtchinnikov, M. and Easter, R. C.: Nonlinear Advection Algorithms Applied to Interrelated Tracers: Errors and Implications for Modeling Aerosol-Cloud Interactions, Mon. Weather Rev., 137, 632-644, doi:10.1175/2008MWR2626.1, 2009.

Prather, M.: Numerical advection by conservation of second-order moments, J. Geophys. Res., 91, 6671-6681, 1986.

Thuburn, J. and Mclntyre, M.: Numerical advection schemes, crossisentropic random walks, and correlations between chemical species, J. Geophys. Res., 102, 6775-6797, 1997.

Ullrich, P. A., Lauritzen, P. H., and Jablonowski, C.: Some considerations for high-order "incremental remap"-based transport schemes: edges, reconstructions and area integration, Int. J. Numer. Meth. Fluids, in press, doi:10.1002/fld.3703, 2012.

Williamson, D. L., Drake, J. B., Hack, J. J., Jakob, R., and Swarztrauber, P. N.: A Standard Test Set for Numerical Approximations to the Shallow Water Equations in Spherical Geometry, J. Comput. Phys., 102, 211-224, 1992.

Zalesak, S. T.: Fully multidimensional flux-corrected transport algorithms for fluids, J. Comput. Phys., 31, 335-362, 1979.

Zerroukat, M., Wood, N., and Staniforth, A.: SLICE: A SemiLagrangian Inherently Conserving and Efficient scheme for transport problems, Q. J. R. Meteorol. Soc., 128, 2801-2820, 2002. 